\title{
TG2 transamidating activity acts as a reostat controlling the interplay between apoptosis and autophagy
}

\author{
Federica Rossin • Manuela D'Eletto • \\ Douglas Macdonald • Maria Grazia Farrace • \\ Mauro Piacentini
}

Received: 23 December 2010/Accepted: 22 March 2011/Published online: 10 April 2011

(C) Springer-Verlag 2011

\begin{abstract}
Tissue transglutaminase (TG2) activity has been implicated in inflammatory disease processes such as Celiac disease, infectious diseases, cancer, and neurodegenerative diseases, such as Huntington's disease. Furthermore, four distinct biochemical activities have been described for TG2 including protein crosslinking via transamidation, GTPase, kinase and protein disulfide isomerase activities. Although the enzyme plays a complex role in the regulation of cell death and autophagy, the molecular mechanisms and the putative biochemical activity involved in each is unclear. Therefore, the goal of the present study was to determine how TG2 modulates autophagy and/or apoptosis and which of its biochemical activities is involved in those processes. To address this question, immortalized embryonic fibroblasts obtained from TG2 knock-out mice were reconstituted with either wild-type TG2 or TG2 lacking its transamidating activity and these were subjected to different treatments to induce autophagy or apoptosis. We found that knock out of the endogenous TG2 resulted in a
\end{abstract}

F. Rossin and M. D'Eletto contributed equally to this work.

F. Rossin - M. D’Eletto - M. G. Farrace · M. Piacentini ( $₫)$

Department of Biology, University of Rome "Tor Vergata",

Via della Ricerca Scientifica 1, 00133 Rome, Italy

e-mail: mauro.piacentini@uniroma2.it

\section{Piacentini}

National Institute for Infectious Diseases IRCCS "Lazzaro

Spallanzani”, 00149 Rome, Italy

D. Macdonald

CHDI Management/CHDI Foundation,

Los Angeles, CA, USA significant exacerbation of caspase 3 activity and PARP cleavage in MEF cells subjected to apoptotic stimuli. Interestingly, the same cells showed the accumulation of LC3 II isoform following autophagy induction. These findings strongly suggest that TG2 transamidating activity plays a protective role in the response of MEF cells to death stimuli, because the expression of the wild-type TG2, but not its transamidation inactive C277S mutant, resulted in a suppression of caspase 3 as well as PARP cleavage upon apoptosis induction. Additionally, the same mutant was unable to catalyze the final steps in autophagosome formation during autophagy. Our findings clearly indicate that the TG2 transamidating activity is the primary biochemical function involved in the physiological regulation of both apoptosis and autophagy. These data also indicate that TG2 is a key regulator of cross-talk between autophagy and apoptosis.

Keywords Transglutaminase 2 - Apoptosis - Autophagy · Transamidating activity

\section{Introduction}

Transglutaminases (EC 2.3.2.13; TG) are a family of enzymes that catalyse thiol- and $\mathrm{Ca}^{2+}$-dependent transamidation reactions, through the formation of a covalent bond between the $\gamma$-carboxamide group of a peptide bond glutamine residue and a primary amine group (Folk and Finlayson 1977). The most ubiquitous isoenzyme is represented by type 2 transglutaminase (TG2), which has been found both intracellularly as well as on the cell surface in association with the extra-cellular matrix (Gaudry et al. 1999; Piacentini et al. 2002). TG2 has been implicated in a variety of cellular processes, such as differentiation, cell 
death, inflammation, cell migration, and wound healing (Collighan and Griffin 2009; Fesus and Piacentini 2002; Fesus and Szondy 2005; Lorand and Graham 2003).

The vast array of biochemical functions catalysed by TG2 distinguishes it from the other members of the TG family. In fact, in addition to its transamidating activity, TG2 might also act as a GTP-binding protein that mediates intracellular signaling by coupling several receptors to the phospholipase C-gamma1 (Fesus and Piacentini 2002). This activity is inhibited by $\mathrm{Ca}^{2+}$, which acts as a switch between the two main biochemical functions of the enzyme (Melino and Piacentini 1998). More recent evidence indicates that under physiological circumstances the TG2 enzyme may also act as protein disulfide isomerase (PDI) (Hasegawa et al. 2003; Mastroberardino et al. 2006) and as a serine/threonine kinase (Mishra and Murphy 2004).

Importantly, all members of the TG superfamily possess a catalytic triad of cysteine 277 (C277), histidine 335 (H335) and aspartate 358 (D358), which is requisite for transamidating activity (Liu et al. 2002; Pedersen et al. 1994; Yee et al. 1994). The cysteine to serine mutation at the position 277 (C277S) has been extensively used to inactivate the transamidation function of TG2 (Tucholski and Johnson 2002). Furthermore, the various biochemical activities of TG2 are differentially regulated depending on its subcellular localization and may exert differential effects on cell survival (Antonyak et al. 2006; Mehta et al. 2006).

One of the primary roles that TG2 plays in the cell is the modulation of the cell death processes. Depending on cell type and apoptotic stimuli, TG2 can exert a protective role (Cao et al. 2008; Yamaguchi and Wang 2006), as well as a facilitatory role, on apoptosis (Datta et al. 2007; Fesus and Szondy 2005; Piacentini et al. 2002; Szondy et al. 2006; Tucholski and Johnson 2002). Recently, it has also been shown that TG2 is involved in the later stages of maturation of autophagolysosomes (D'Eletto et al. 2009).

A growing number of publications demonstrate that apoptosis induction is often associated with increased autophagy, indicating the existence of an interplay between these two important cellular events (Fimia and Piacentini 2010). It is becoming clear that autophagy may contribute differently to cell death induction depending on the type and degree of environmental changes, or stress stimuli, as well as the cell type. Inhibition of autophagy leads in most cases to an increased susceptibility to apoptotic stimuli, highlighting the pro-survival role of the autophagic process (Eisenberg-Lerner et al. 2009; Fimia and Piacentini 2010; Yousefi et al. 2006).

Deregulation of autophagy has been proposed to play a major role in several pathologies, including cardiomyopathy, hepatitis, diabetes and neurodegenerative disorders
(Facchiano et al. 2006; Levine and Kroemer 2008; Rubinsztein et al. 2007), which are all diseases where the involvement of TG2 has been clearly established (Kahlem et al.1998; Malorni et al. 2008; Mastroberardino et al. 2002).

Although the involvement of TG2 in apoptosis and autophagy is well documented, the molecular mechanisms of its action on these processes is still unknown. In particular, it is still not fully established which of the several TG2 biochemical activities is involved. Therefore, the goal of the present study was to dissect out the biochemical and cellular mechanisms involved in either the autophagic or apoptotic activity of TG2. To this aim immortalized fibroblasts obtained by TG2 knock-out mouse embryos stably transfected with active wild-type TG2 or TG2 without transamidating activity (C277S) were used to determine how TG2 modulates apoptosis and autophagy in response to different treatments.

\section{Materials and methods}

\section{Materials}

Anti-tubulin (T-4026) and anti-actin (A-2066) were from Sigma, anti-LC3 (NB100-2331) was from Novus Biologicals, mouse monoclonal TG2 antibody TG100 (MS279-P) or CUB7402 (MS-224-P1) were from Lab Vision NeoMarkers, anti-PARP (SA-250) was from Biomol, anti-caspase 3 (9662) was from Cell Signaling and antiANT (AP 1034) was from Calbiochem. Secondary antibodies were from Jackson Immunoresearch Labs. Earle's balanced salt solution (EBSS), 2-deoxy-D-glucose (2-DG), Staurosporine (STS), $\mathrm{NH}_{4} \mathrm{Cl}$ (A0171), acridine orange and puromycin were obtained from Sigma, D-MEM and FBS were from Invitrogen Life Technologies Inc., ECL detection system (Chemi-Glow) was from Alpha Innotech, 5-(biotinamido)pentylamine and horseradish peroxidase (HRP)-conjugated streptavidin were from Pierce.

\section{Cloning of TGase 2 gene}

TG2 cDNA was obtained by polymerase chain reaction (PCR) amplification using a human fibroblast cDNA library (Clontech, Palo Alto, CA, USA). The construct of wt human TG2 gene was designed to be inserted into the XhoI and EcoRI sites of the pLPCX vector obtaining the pLPCX-TG2 plasmid. The full length human TG2 gene was ligated into the XhoI and EcoRI sites of the pLPCX vector, obtaining the pLPCX-TG2 plasmid, which was transformed and sequenced to verify the amplification efficacy. The mutant of the plasmid pLPCX-TG2 was 
obtained using Quick Change Site-Directed Mutagenesis kit (Stratagene).

The primers used for mutant of the plasmid pLPCXTG2 in the cysteine 277 (pLPCX-C277S) were:

C277S 5'-tcaagtatggccagagctgggtcttcgcc- $3^{\prime}$

C277S_antisense $5^{\prime}$-ggcgaagacccagctctggccatacttga- $3^{\prime}$

pLPCX-TG2 plasmid was used as mutagenesis template. The PCR-amplification products were treated with restriction enzyme DpnI (Fermentas). An aliquot of $5 \mu$ l above PCR product was transformed into DH $5 \alpha$ competent cells and inoculated on Luria-Bertani (LB) plate containing $100 \mu \mathrm{g} / \mathrm{ml}$ ampicillin. A total of 10 colonies were selected and their plasmids were isolated by mini-prep. The positive mutants were selected by DNA sequencing. pLPCX plasmid contains a packaging signal under the control of a Moloney murine leukemia virus $5^{\prime}$ long terminal repeat and a puromycin resistance gene under the control of the CMV promoter.

TG2 knockout reconstitution by retrovirus infection

pLPCX-TG2 and pLPCX-C277S were transfected in the Bosc 23 ecotropic packaging line using $\mathrm{CaCl}_{2}$ and $\mathrm{HBS}$ to create high titer of ecotropic retrovirus. After $48 \mathrm{~h}$, the retroviral supernatant was harvested, filtered through a $0.45 \mu \mathrm{m}$ membrane and used to transduce -/-MEF cells. For the creation of cell lines, -/-MEF cells were transduced by replacing the culture medium with retroviral conditioned medium from Bosc 23 ecotropic packaging cells, in the presence of $8 \mu \mathrm{g} / \mathrm{ml}$ Polybrene (Sigma) followed by incubation for $16-18 \mathrm{~h}$ at $37^{\circ} \mathrm{C}$. The culture medium was replaced with fresh D-MEM, and cells were grown, trypsinized, replated, and selected for puromycin resistance using the antibiotic added to the culture medium $(3 \mu \mathrm{g} / \mathrm{ml})$. We had previously performed a survival curve with the antibiotic on untransfected cells to determine optimal antibiotic concentration and plating density.

Cell culture and treatments

MEF cells were cultured in Dulbecco's modified Eagle's medium (D-MEM) supplemented with 10\% fetal bovine serum, $2 \mathrm{mM}$ L-glutamine, $100 \mathrm{mg} / \mathrm{ml}$ streptomycin and 100 units $/ \mathrm{ml}$ penicillin. Cells were grown in a humidified atmosphere containing $5 \% \mathrm{CO}_{2}$ at $37^{\circ} \mathrm{C}$. For amino acid starvation (STV), cells were washed two times in Earle's balanced salt solution (EBSS) and incubated in EBSS for the indicated periods in presence or not of $20 \mathrm{mM} \mathrm{NH}_{4} \mathrm{Cl}$. For apoptosis induction, MEF cells were treated with $2 \mu \mathrm{M}$ STS for $4 \mathrm{~h}$, or with $10 \mathrm{mM}$ 2-deoxy-D-glucose (2-DG) for $36 \mathrm{~h}$ at $37^{\circ} \mathrm{C}$.
Preparation of isolated mitochondria

Mitochondria were prepared by differential centrifugation: unbroken cells, nuclei and large membranes were removed through a centrifugation at $500 \mathrm{~g}$ for $15 \mathrm{~min}$. The supernatant was further centrifugated at $9,000 \mathrm{~g}$ for $20 \mathrm{~min}$. The mitochondrial enriched fraction, was resuspended in $50 \mathrm{mM}$ Tris- $\mathrm{HCl} \mathrm{pH} 7.4,1 \mathrm{mM}$ EDTA with protease inhibitor. Proteins were quantified with standard Bradford staining.

Immunoblotting

Cells were rinsed in ice-cold phosphate-buffered saline (PBS) and collected in lysis buffer, containing $20 \mathrm{mM}$ Tris-HCl pH 7.4, $150 \mathrm{mM} \mathrm{NaCl}, 1 \%$ Triton X-100 with protease inhibitor. Samples were centrifuged at $9,000 \mathrm{~g}$ for 15 min. Protein concentrations were determined by the Bradford assay, using bovine serum albumin as a standard. Aliquots of total protein extracts $(30 \mu \mathrm{g})$ from cells after different treatments were resolved on a $12 \%$ pre-cast SDSpolyacrylamide gel (Invitrogen) and transferred to nitrocellulose membrane. Blots were blocked in 5\% nonfat dry milk in T-PBS (PBS $+0.05 \%$ Tween 20 ) for $1 \mathrm{~h}$ at room temperature. The blots were then incubated overnight with above described antibodies. The membranes were washed three times with T-PBS and incubated with HRP-conjugated secondary antibody for $1 \mathrm{~h}$ at room temperature. The membranes were rinsed three times with T-PBS, and the signal was detected by enhanced ECL chemiluminescent detection system.

In situ transglutaminase assay

For in situ TG2 activity measurements, the cells were preincubated for $20 \mathrm{~min}$ with $2 \mathrm{mM} \mathrm{5}$-(biotinamido)pentylamine, prior to treatment with $2 \mu \mathrm{M}$ STS for $4 \mathrm{~h}$. Cells were then harvested, lysed as described above and incorporation of the reagent into proteins was determined by blotting with HRP-conjugated streptavidin $(1: 2,000)$. The blots were then developed following the standard ECL protocol.

\section{Fluorescence microscopy}

Sub-confluent cells (MEFs) grown on coverlips were transiently transfected with pLPCX-GFP-LC3 vector using lipofectamineTM 2000 (Invitrogen) according to the manufacturer's protocols. Acidic compartments were labeled by incubating the cells with $75 \mathrm{nM}$ LysoTracker Red (Molecular Probes) in the culture medium at $37^{\circ} \mathrm{C}$ for $20 \mathrm{~min}$. Nuclei were stained with $10 \mu \mathrm{g} / \mathrm{ml}$ Hoechst 33342 for $20 \mathrm{~min}$. Cells were then fixed with $4 \%$ 
paraformaldehyde for $20 \mathrm{~min}$ at room temperature. The coverslips were mounted on microscope slides, sealed with an antifade solution and examined with a image workstation DeltaVision (AppliedPrecision) Olympus 1 X70 microscope.

\section{Analysis by FACS}

To evaluate the acidification of the lysosomal compartment, cells were incubated with $1 \mu \mathrm{g} / \mathrm{ml}$ Acridine Orange for $20 \mathrm{~min}$ at room temperature, with protection from light. Cells, treated as above described, were analysed on a FACScan (Becton-Dickinson) cytometer. CellQuest software was used for analysis.

Data analysis

All the data reported were verified in at least three different experiments and reported as mean \pm SEM. Only $p<0.01$ were considered significant.

\section{Results}

Characterization of the TG2 knockout MEFs reconstituted with the wild type and $\mathrm{C} 277 \mathrm{~S}$ mutant TG2 enzyme

To determine the role of TG2 in autophagy or apoptosis, we expressed the wild-type TG2 or the enzyme mutated at cysteine 277 (C277S), which is the active site for transamidating activity, into immortalized fibroblasts obtained from TG2 knock-out mouse embryos (-/-MEF) (D'Eletto et al. 2009).

We stably transfected -/-MEFs by retroviral infection, and after antibiotic selection with puromycin $(3 \mu \mathrm{g} / \mathrm{ml})$, we isolated various independent puromycin resistant clones from the -/-MEF-transduced cells in which the TG2 expression was detected by western blot analysis as compared to -/-MEFs. In Fig. 1, the level of TG2 expression of three cell clones expressing wild-type TG2 $\left(-/-\mathrm{MEF}^{\mathrm{WT}} 1, \quad-/-\mathrm{MEF}^{\mathrm{WT}} 2, \quad-/-\mathrm{MEF}^{\mathrm{WT}} 3\right.$; $\quad$ Fig. 1a) and the TG2 inactive $\mathrm{C} 277 \mathrm{~S}$ mutant $\left(-/-\mathrm{MEF}^{\mathrm{C} 277 \mathrm{~S}} \mathrm{~A}\right.$, $-/-\mathrm{MEF}^{\mathrm{C} 277 \mathrm{~S}} \mathrm{~B},-/-\mathrm{MEF}^{\mathrm{C} 277 \mathrm{~S}} \mathrm{C}$; Fig. 1b) is reported. These cell clones were choosen since they expressed protein levels comparable to the wild-type MEFs to study the effect of TG2 transamidating activity on induction of apoptosis and autophagy in response to different stimuli.

In a first set of experiments we used the $5^{\prime}$-(biotinamido)pentylamine incorporation method to verify whether the reconstituted cell clones (either the $-/-\mathrm{MEF}^{\mathrm{WT}}$ and $-/-\mathrm{MEF}^{\mathrm{C} 277 \mathrm{~S}}$ ) display the expected transamidating-TG2 activity. Intracellular TG2 is known to be largely inactive under normal physiological condition, but it is activated under stressful conditions. In keeping with this, we treated the above-mentioned clones with STS, a well-known inductor of apoptosis. As shown in Fig. 1c we found that intracellular TG2 activity upon apoptosis induction by STS increased both in +/+MEF and in reconstituted $-/-\mathrm{MEF}^{\mathrm{WT}}$ cells, but was effectively suppressed in $-/-\mathrm{MEF}$ and $-/-\mathrm{MEF}^{\mathrm{C} 277 \mathrm{~S}}$ cells, as expected due to its active-site inactivation. To further characterize the selected cell clones we also verified the subcellular localization of the reconstituted TG2 by isolating cytosolic and mitochondrial fractions from transfected cells as well as from $2 \mathrm{~F}$ TGH as a control cell line expressing high levels of endogenous TG2 protein. The purity of cytosolic and mitochondrial fractions was verified by immunoblotting for adenine nucleotide translocator (ANT, as a resident mitochondrial protein) and tubulin (as a cytosolic protein) (Fig. 1d). As expected, the majority of TG2 protein was localized in the cytoplasm although an appreciable amount of TG2 was also detected in the mitochondrial fractions of the transfected cells (Fig. 1d). Notably, the active-site mutation did not cause any change in the subcellular localization of TG2 (Fig. 1d).

Effect of TG2 transamidating activity on apoptosis induction

It has been well established in different experimental systems that TG2 is one of the effector genes induced in cells undergoing apoptosis in vivo (Oliverio et al. 1999; Piacentini et al. 2002; Tucholski and Johnson 2002). Previous studies suggest that TG2 can either facilitate or prevent apoptosis depending on the cell type, the kind of stimuli, the cellular localization or the prevalence of the different enzyme activities (Antonyak et al. 2006; Cao et al. 2008; Mehta et al. 2006; Piacentini et al. 1991; Tucholski and Johnson 2002).

In order to clarify the involvement of TG2 transamidating activity in cell death, +/+MEF, -/-MEF, $-/-\mathrm{MEF}^{\mathrm{WT}}$ and $-/-\mathrm{MEF}^{\mathrm{C} 277 \mathrm{~S}}$ were treated with the potent apoptotic stimulus, STS. Apoptosis was assessed by western blot analysis using the specific apoptotic markers of caspases 3 activation and PARP cleavage. As shown in Fig. 2a, a marked induction of PARP cleavage and caspase 3 activation was seen in -/-MEFs when compared with $+/+$ MEFs. Furthermore, when the wild-type TG2 was reconstituted in $-/-$ MEFs, they displayed a protective effect against death stimuli, as demonstrated by the absence of both PARP cleavage and caspase 3 activation after STS treatment (Fig. 2b). Interestingly, the contrary was observed in the $-/-\mathrm{MEF}^{\mathrm{C} 277 \mathrm{~S}}$ clones lacking the enzyme's transamidating activity, which resulted in an increased sensitivity to STS when compared with $+/+\mathrm{MEF}$ 
a

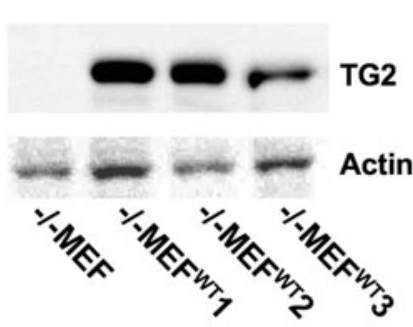

C STS

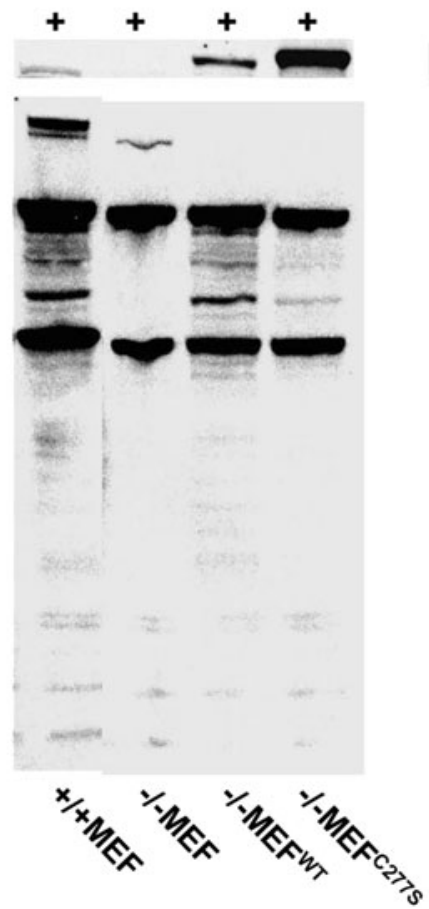

Fig. 1 Expression of TG2 in -/-MEF cells stably transfected with TG2 constructs. Detection of TG2 expression levels in cell lysates from three cell clones expressing a wild-type TG2 construct and b C277S mutant. Actin was used to verify protein loading. c Transamidating activity of TG2 in the MEF cells treated with STS. Representative blot of the TG-catalyzed incorporation of 5-(biotinamido)pentylamine into proteins as a measure of in situ enzyme activity. Cells were treated with $2 \mu \mathrm{M}$ STS for $2 \mathrm{~h}$ and labeled with 5-(biotinamido)pentylamine. Blots probed with HRPconjugated streptavidin indicate that TG2 activity upon apoptosis

and $-/-\mathrm{MEF}^{\mathrm{WT}}$ cells (Fig. 2b). It is important to mention that we have isolated and analysed more than 15 different clones for the wild-type enzyme and 6 for the $\mathrm{C} 277$ mutant. Although they present some quantitative differences as shown in Fig. $2 b$ they all share the same behavior as far as the apoptosis induction is concerned.

In order to confirm the differential response of $-/-\mathrm{MEF}^{\mathrm{WT}}$ and $-/-\mathrm{MEF}^{\mathrm{C} 277 \mathrm{~S}}$ cells to apoptosis induction, we also compared their sensitivity to 2-deoxy-D-glucose (2-DG), a non-metabolisable analog of glucose. 2-DG has been shown to inhibit glycolysis by depleting the cell's ATP content by $25-30 \%$, leading to the induction of b

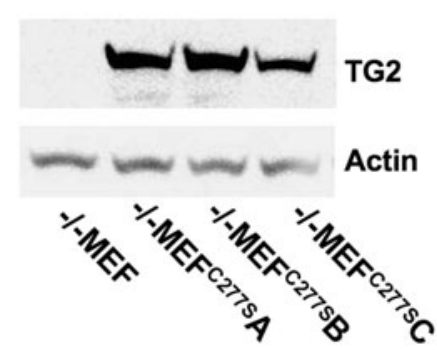

d

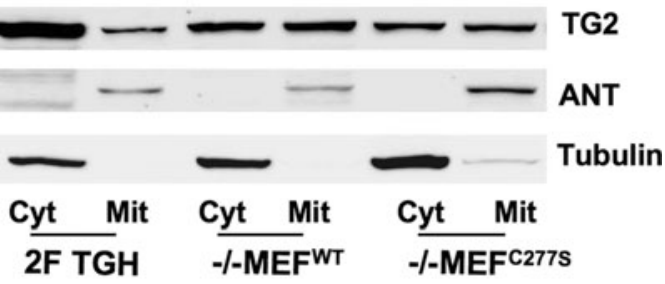

induction increased both in $+/+\mathrm{MEF}$ and $-/-\mathrm{MEF}^{\mathrm{WT}}$ cells compared to either $-/-\mathrm{MEF}$ or $-/-\mathrm{MEF}^{\mathrm{C} 277 \mathrm{~S}}$, according with the active-site inactivation. d Subcellular localization of TG2. Cells were separated into cytosolic and mitochondrial fractions and their TG2 levels were analysed by western blot. 2F TGH cells were used as control of subcellular localization of human TG2. TG2 blot shows that the clone reconstitution has no significant effect on the enzyme intracellular localization. ANT (mitochondrial marker) and tubulin (cytosolic marker) were used to verify the purity of the fractions

autophagy and/or apoptosis depending on the cell type (D'Eletto et al. 2009). Here, we treated the cell clones with $10 \mathrm{mM}$ 2-DG for $36 \mathrm{~h}$ and assessed the induction of apoptosis by caspases 3 activation and PARP cleavage. As shown in Fig. 2c, PARP cleavage increased significantly after 2-DG treatment in $-/-\mathrm{MEF}$ and in the $-/-\mathrm{MEF}^{\mathrm{C} 277 \mathrm{~S}}$ cells. In contrast, no significant increase in PARP cleavage was detected in either the $+/+\mathrm{MEF}$ or $-/-\mathrm{MEF}^{\mathrm{WT}}$ cells. These results were confirmed by the activation of caspase 3 , which appeared only in -/-MEF and $-/-\mathrm{MEF}^{\mathrm{C} 277 \mathrm{~S}}$ cells (Fig. 2c), thus substantiating the protective role exerted by TG2 in the cells through its transamidating activity. 


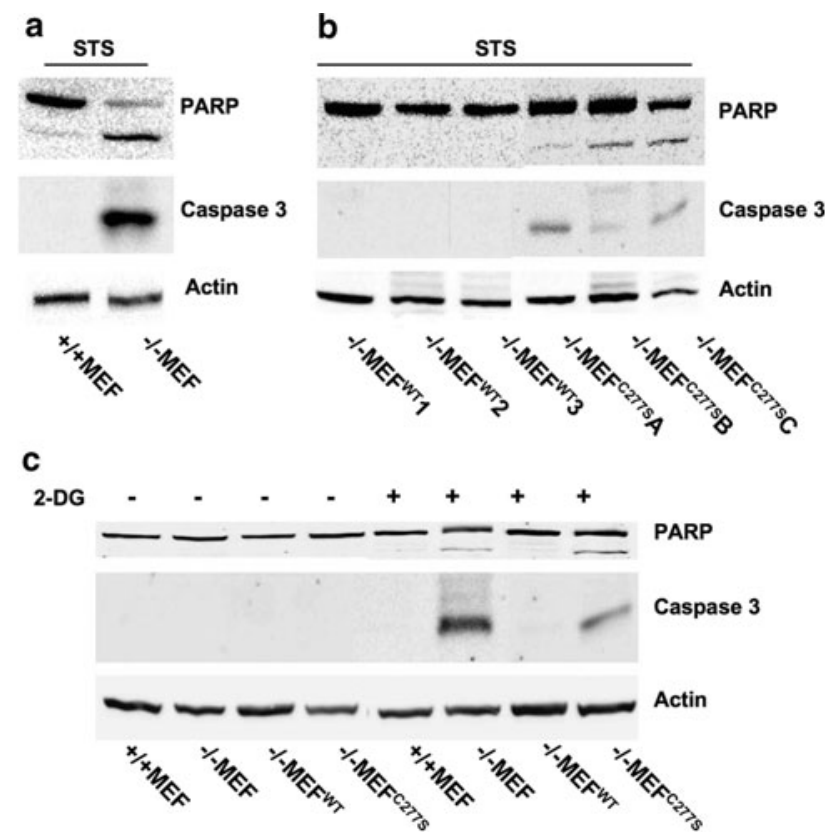

Fig. 2 Wild-type TG2 protects against STS induced apoptosis in MEF cells. a, b Cell lines were treated with STS $(2 \mu \mathrm{M})$ for $4 \mathrm{~h}$ and cell lysates were subjected to Western blot analysis with anti-PARP and anti-Caspase 3 to examine apoptosis induction. a The active form of caspase 3 and the PARP cleavage appear only in -/-MEF as compared to $+/+\mathrm{MEF}$ cells. b Similar results were detected in -/-MEF cells expressing C277S mutant as compared to wild-type TG2. c Cells were incubated in full medium in the presence of $10 \mathrm{mM}$ 2-DG for $36 \mathrm{~h}$. Then they were lysed and proteins subjected to immunoblotting for expression of the above indicated antibodies. PARP cleavage and caspase 3 activation, increased both in $-/-\mathrm{MEF}$ and $-/-\mathrm{MEF}^{\mathrm{C} 277 \mathrm{~S}}$ cells compared to either $+/+\mathrm{MEF}$ or $-/-\mathrm{MEF}^{\mathrm{WT}}$, according with the active-site inactivation

Effect of TG2 transamidating activity on autophagy induction

In previous studies, we have demonstrated a role for TG2mediated post-translational modifications of proteins in the final maturation of autophagolysosomes under physiological conditions (D'Eletto et al. 2009). In fact, the lack of TG2 leads to an impairment of the autophagosome formation and LC3 II accumulation both in knock out isolated MEFs and in mouse tissues (D'Eletto et al. 2009). In order to define whether the TG2-transamidating activity is involved in autophagic process we first analyzed the effect of nutrient depletion on the $+/+$ MEFs and $-/-$ MEFs incubated in an amino acid-free and serum-free medium for $18 \mathrm{~h}$. Autophagy was monitored by detecting the conversion of LC3 I into LC3 II by western blot analysis. As reported in Fig. 3a, the levels of the $16 \mathrm{kDa}$ LC3 II was increased in $-/-\mathrm{MEFs}$ as compared to the $+/+\mathrm{MEFs}$, confirming the impairment of the autophagosomes maturation observed in absence of TG2. Interestingly, all of the

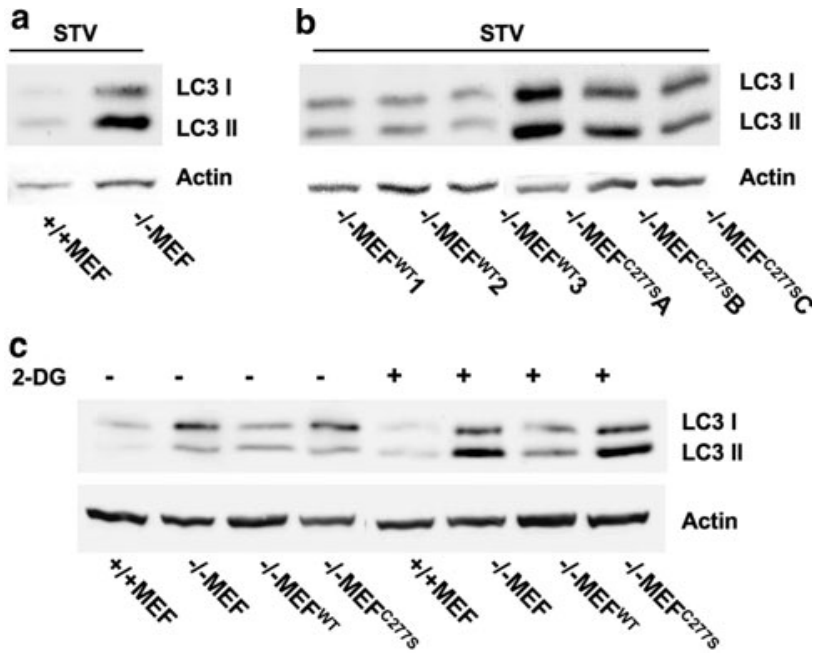

Fig. 3 Effect of autophagy induction on TG2 reconstituted MEF cell lines. Cells cultured in an amino acid-free and serum-free medium (EBSS) were collected after $18 \mathrm{~h}$, lysed and proteins were subjected to immunoblotting for expression of LC3, to examine autophagy induction. a Note the evident accumulation of LC3 II isoform in $-/-$ MEF but not in +/+MEF cells. b LC3 II is significantly decreased in $-/-\mathrm{MEF}$ cells expressing wild-type TG2 compared to $\mathrm{C} 277 \mathrm{~S}$, indicating that stable reconstitution of $-/-\mathrm{MEF}$ with wildtype TG2 restores the capacity of these cells to complete the autophagic process. c Cells were incubated in full medium in the presence of $10 \mathrm{mM}$ 2-DG for $36 \mathrm{~h}$ and subsequently lysed and proteins subjected to immunoblotting for expression of LC3. LC3 II isoform accumulation increased both in $-/-\mathrm{MEF}$ and $-/-\mathrm{MEF}^{\mathrm{C} 277 \mathrm{~S}}$ cells compared to either $+/+\mathrm{MEF}$ or $-/-\mathrm{MEF}^{\mathrm{WT}}$, according to the active-site inactivation

analyzed WT TG2 reconstituted cell clones showed an evident decrease in the LC3 II accumulation as compared to the -/-MEF cells (Fig. 3b) indicating that the re-introduction of TG2 protein into the $-/-$ MEFs rescued the normal autophagyc flux. On the contrary, there was an evident accumulation of LC3 II in all of the C277S mutant cell clones $\left(-/-\mathrm{MEF}^{\mathrm{C} 277 \mathrm{~S}} \mathrm{~A},-/-\mathrm{MEF}^{\mathrm{C} 277 \mathrm{~S}} \mathrm{~B}\right.$, $\left.-/-\mathrm{MEF}^{\mathrm{C} 277 \mathrm{~S}} \mathrm{C}\right)$ when compared with $+/+\mathrm{MEFs}$ (Fig. 3b), indicating that the impairment in the maturation of autophagosomes, observed in the absence of TG2, strictly depends on its transamidating activity (D'Eletto et al. 2009). Similar results were obtained using a different autophagic stimulus such as $10 \mathrm{mM}$ of 2-DG for $36 \mathrm{~h}$ (Fig. 3c).

Next we analyzed whether the lack of TG2-transamidating activity affects the final steps in the autophagosomes maturation, by transiently transfecting the MEF cell clones with GFP-LC3 and staining with LysoTracker Red (LTR). Autophagy was induced by STV in the presence of $\mathrm{NH}_{4} \mathrm{Cl}$, which is known to block lysosomal degradation and thus leading to a marked accumulation of autophagolysosomes. Results reported in Fig. 4a clearly indicate the presence of an elevated number of autophagolysosomes in $+/+$ MEFs as well as in $-/-\mathrm{MEF}^{\mathrm{WT}}$ cells. By contrast, a defect in the 

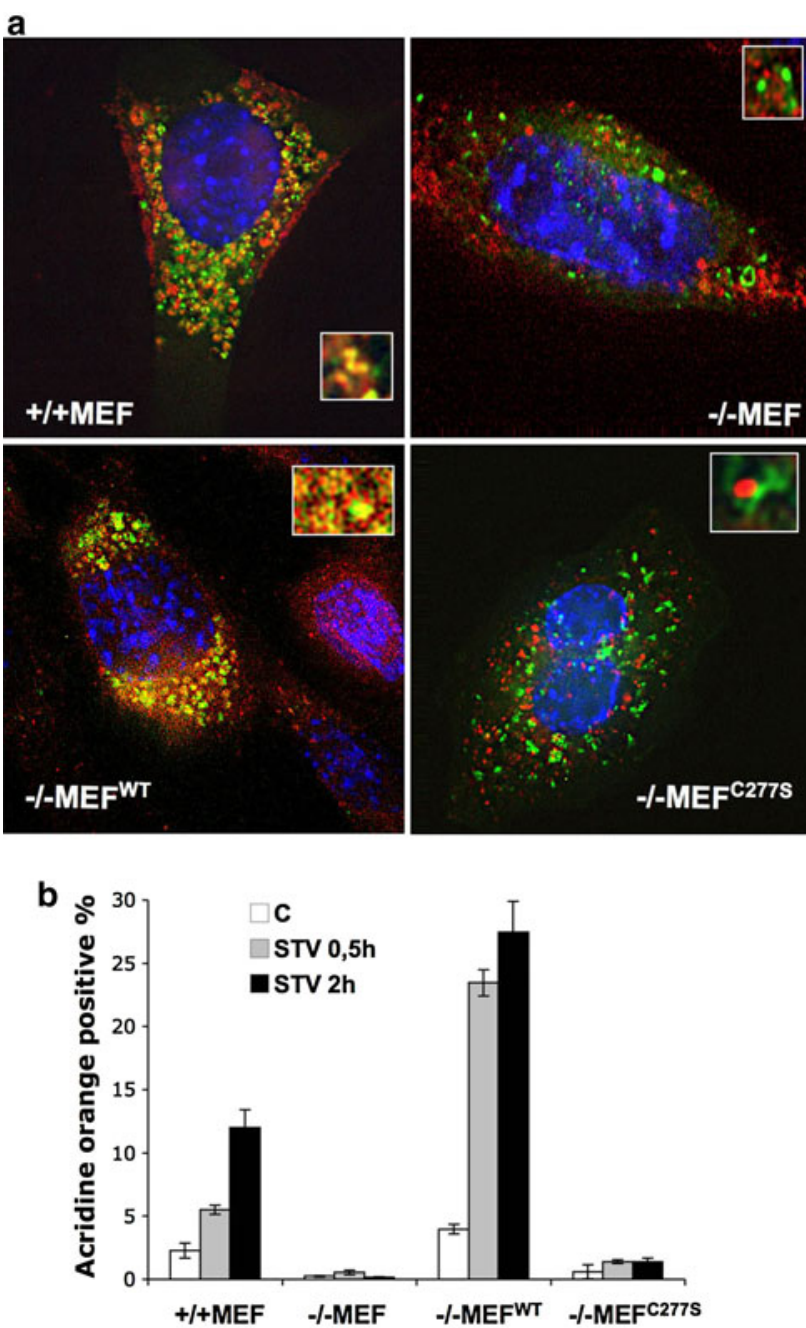

Fig. 4 Detection of acidic vesicular organelles in wild type versus $-/$ MEF reconstituted cell clones. a Immunofluorescence analysis in MEF cell lines undergoing autophagy in the presence of $\mathrm{NH}_{4} \mathrm{Cl}$, which inhibits the activation of the lysosomal enzymes, hence blocking the degradation process of LC3 II isoform. MEF cells were transiently transfected with pLPCX-GFP-LC3 (green), acid compartments were labeled by incubating cells with $75 \mathrm{nM}$ LTR (red) and nuclei were stained with $10 \mu \mathrm{g} / \mathrm{ml}$ Hoechst 33342 (blue) for $20 \mathrm{~min}$. Green dots represent autophagosomes; red dots represent free lysosomes; yellow dots are sites of overlap of GFP-LC3 with LTR and highlight the formation of autophagolysosomes. b Quantification of acidic vesicular organelles in MEFs by acridine orange staining using FACS analysis. Cells were subjected to STV, incubated with Acridine Orange and then analyzed on a FACScan cytometer. The increased acidification of autophagosomes in the $+/+\mathrm{MEF}$ and in $-/-\mathrm{MEF}^{\mathrm{WT}}$ cells demonstrate that the reconstitution of $-/-\mathrm{MEF}$ with wild-type TG2, but not with $\mathrm{C} 277 \mathrm{~S}$ mutant, restores the capacity of these cells to complete the autophagic process. Values are means \pm SEM of 3-4 determinations

fusion of autophagosomes with lysosomes was detected in the cells lacking TG2-transamidating activity (-/-MEFs and $-/-\mathrm{MEF}^{\mathrm{C} 277 \mathrm{~S}}$ ), as highlighted by the absence of overlap of GFP-LC3 with LysoTracker Red.
It is well-known that during autophagy, the acidification of the autophagolysosome's lumen enables this specialized membrane system to degrade sequestered cytoplasmic components (D'Eletto et al. 2009). To study the effect of TG2-transamidating activity on the maturation of autophagolysosomes, we used the lysosomotropic agent acridine orange, a weak base that moves freely across biological membranes when uncharged which accumulates in acidic compartments where it forms red fluorescent aggregates that can be detected by flow cytometry.

As expected, the acidification of autophagosomes increased after STV in the $+/+\mathrm{MEF}$ and in $-/-\mathrm{MEF}^{\mathrm{WT}}$ cells, while the expression of the active-site mutated TG2 failed to restore the capacity of these cells to complete the autophagic process, resulting in the absence of acidic vesicular organelles detected by acridine orange staining (Fig. 4b). These studies demonstrate that the TG2-transamidating activity is sufficient and necessary to catalyze the final steps of autophagosome formation.

\section{Discussion}

The balance between cell proliferation and death allows multi-cellular organisms to model their shape during development and to maintain their own homeostasis in adulthood. A large amount of evidences show that apoptosis induction is often associated with a concomitant modulation of autophagy indicating the existence of an interplay between these two important cellular events (Fimia and Piacentini 2010; Klionsky 2007; Maiuri et al. 2007b). The simultaneous regulation of both mechanisms has been detected not only in experimental settings, but also in vivo under physiological and pathological conditions. The best evidence that the regulation of autophagy and apoptosis are strictly interconnected is highlighted by the fact that the two pathways share key molecular regulators (Eisenberg-Lerner et al. 2009). Of particular note is that TG2 protein levels and its activity have been shown to be induced in both phenomena. However, the role that TG2 plays on the two pathways has not been completely understood. In keeping with this assumption, in this study we focused our attention on the role of TG2 and its transamidation enzymatic activity on the regulation of apoptosis and autophagy in mouse fibroblasts.

It is well established that intracellular TG2 is able to carry out three different enzymatic activities in addition to its predominant transamidase and deamidase activities. These functions are: (i) the GTPase activity and its related intra-cellular $G$ protein signalling, via coupling to the $\alpha_{1 \mathrm{~b}} / \alpha_{1 \mathrm{~d}}$-adrenergic receptors (Nakaoka et al. 1994), the TP $\alpha$ thromboxane A2 receptor (Vezza et al. 1999), and the oxytocin receptor (Park et al. 1998); (ii) a serine/threonine 
kinase activity, (Mishra and Murphy 2004); (iii) a PDI activity (Hasegawa et al. 2003; Mastroberardino et al. 2006) for which several mitochondrial substrates have been identified (Malorni et al. 2009; Piacentini et al. 2002). Under stressful conditions, such as those induced by many cell death and authophagic stimuli, where the free intracellular calcium concentrations become elevated, TG2 becomes a cross-linking enzyme (Di Venere et al. 2000). This is in contrast to its structure when bound to GDP which have been resolved in a crystal complex (Liu et al. 2002). In that GDP-bound conformation, four distinct domains were identified: a $\mathrm{N}$-terminal $\beta$-sandwich, bearing fibronectin and integrin-binding sites; a catalytic core, bearing the catalytic triad for the acyl-transfer reaction and a putative $\mathrm{BH} 3$ domain; and finally, the two C-terminal $\beta$-barrel domains. The binding of GTP, but not that of $\mathrm{Ca}^{2+}$, proves to be important for the stability of the enzyme's conformation, suggesting a molecular mechanism by which GTP inhibits TG2-transamidation activity. Accordingly, the GTP-TG2 adopts a "closed" conformation which decreases the accessibility of the protein matrix to the solvent, thus rendering the accessibility of the cystamine-277 containing active site more hindered (Di Venere et al. 2000).

Under physiological conditions, TG2 over-expression primes cells for suicide while its inhibition, through several strategies, results in decreased cell death (Malorni et al. 2009; Oliverio et al. 1999). It has been reported that the enzyme might sensitize cells towards apoptosis by interacting with mitochondria and by shifting them to a higher polarized state (Malorni et al. 2009; Piacentini et al. 2002). This event triggers an alteration of the redox status that might provoke the activation of the TG2's PDI activity. At the same time, the massive increase of cytosolic $\mathrm{Ca}^{2+}$ concentration, observed during the later stages of apoptosis, determines the switch of TG2 to its cross-linking configuration in all sub-cellular compartments. This transition results in an extensive polymerization of intra-cellular proteins, including actin, $\mathrm{Sp} 1$ and $\mathrm{Rb}$, and the formation of detergent-insoluble structures (Fesus et al. 1989; Oliverio et al. 1999; Tatsukawa et al. 2009). These protein scaffolds stabilize the dying cell before its clearance by phagocytosis; this prevents the release of harmful intra-cellular components and the activation of an inflammatory or autoimmune response (Piacentini and Colizzi 1999). Under pathological conditions, the death of cells expressing high amounts of TG2 may occur as a result of a 'mummification' event caused by an extensive cross-linking of cytosolic proteins without signs of either apoptosis or necrosis.

The results reported in this paper clearly indicate that the TG2 cross-linking activity is the principal enzymatic activity involved in the regulation of both apoptosis and autophagy. In fact, the reconstitution of MEFs, isolated from the TG2-knockout mouse, with either the wild type or the $\mathrm{C} 277 \mathrm{~S}$ inactive mutant, evidenced that the impairment of autophagy and the activation of apoptosis observed in the $-/-$ MEFs could only be recovered by the re-introduction to these cells of the wild-type enzyme and not by its active-site mutant. Our study also indicates that the impairment of the late stages of autophagy observed in the $-/$ MEFs primes the cells for the induction of caspasedependent death. It is interesting to note that there is also an important effect played by the relative TG2 protein level expressed in the cell on the autophagic outcome. In fact, both the absence and the overexpression of TG2 led to an impairment of the autophagic process by acting at different levels in the pathway. We demonstrated both in vitro and in vivo, that the lack of TG2 under normal physiological conditions prevents the formation of mature autophagolysosomes and their acidification, thus impairing the degradation of the substrates (D'Eletto et al. 2009). By contrast, studies carried out in pathological settings (i.e. cystic fibrosis), in which the enzyme is overexpressed, showed that TG2 is able to prevent the induction phase at the level of the endoplasmic reticulum by cross-linking the Beclin1/Ambra1 initiation complex (Luciani et al. 2011). These studies suggest that TG2 is absolutely required for the proper completion of the autophagic process, but its activity must be finely tuned in order to promote the autophagic process. Interestingly, the original concept that inhibition of apoptosis results in autophagic/necrotic cell death is now being extended and it is clear that apoptosis and autophagy can act as partners to induce cell death in a coordinated or cooperative fashion (Fimia and Piacentini 2010). On the other hand, inhibition of autophagy leads, in most cases, to an increase susceptibility to apoptotic stimuli, highlighting the pro-survival role of the autophagic process. It is important to note that Beclin 1-mediated induction of autophagy is inhibited by the antiapoptotic Bcl-2 family members; and interestingly, this interaction involves the binding of $\mathrm{Bcl}-2$ to a $\mathrm{BH} 3$ domain present in Beclin 1 (Maiuri et al. 2007a). Therefore, the proteinprotein interaction network dependent on $\mathrm{BH} 3$ domains present in many pro- and anti-apoptotic proteins does not seem to be exclusive for the regulation of the apoptotic process but it is also involved in the induction of autophagy (Maiuri et al. 2007b). In line with this observation, the BH3-only proteins BAD, BNIP3 and BNIP3-like (BNIP3L), also known as NIX, have been shown to play a role both in cell death and autophagy (Zhang and Ney 2009). We have demonstrated that TG2 contains a putative $\mathrm{BH} 3$ domain by which it can interact with Bcl2 family members and consequently modulating both apoptosis and autophagy (Rodolfo et al. 2004).

Thus, in many physiological settings, autophagy acts by promoting cell survival as an antagonist of apoptosis. In 
this study, we have demonstrated that the ablation of TG2 sensitizes cells for the induction of apoptosis indicating its critical role in balancing the onset of these two essential cellular events. Furthermore, we have identified the transamidation activity of TG2 to be responsible for these actions. These findings indicate TG2 as one of the key elements, such as $\mathrm{p} 53$ and $\mathrm{Bcl} 2$, whose expression and proper activation can determine the cell's fate by inducing cell death or survival.

Acknowledgments We thank Dr P. Mattioli for expert assistance with image processing, and Dr S. Oliverio for help with flow citometry analysis. This work was supported by grants from CHDI Foundation Inc. (USA), Compagnia di San Paolo, and Fondazione Telethon to MP, the Ministry of Health of Italy "Ricerca Corrente" and "Ricerca Finalizzata", and AIRC. The support of the EU grant "Apo-Sys" to MP is also acknowledged.

\section{References}

Antonyak MA, Jansen JM, Miller AM, Ly TK, Endo M, Cerione RA (2006) Two isoforms of tissue transglutaminase mediate opposing cellular fates. Proc Natl Acad Sci USA 103:18609-18614

Cao L, Petrusca DN, Satpathy M, Nakshatri H, Petrache I et al (2008) Tissue transglutaminase protects epithelial ovarian cancer cells from cisplatin-induced apoptosis by promoting cell survival signaling. Carcinogenesis 29:1893-1900

Collighan RJ, Griffin M (2009) Transglutaminase 2 cross-linking of matrix proteins: biological significance and medical applications. Amino Acids 36:659-670

D’Eletto M, Farrace MG, Falasca L, Reali V, Oliverio S, Melino G, Griffin M, Fimia GM, Piacentini M (2009) Transglutaminase 2 is involved in autophagosome maturation. Autophagy 5:1145-1154

Datta S, Antonyak MA, Cerione RA (2007) GTP-binding-defective forms of tissue transglutaminase trigger cell death. Biochemistry 46:14819-14829

Di Venere A, Rossi A, De Matteis F, Rosato N, Agro AF, Mei G (2000) Opposite effects of $\mathrm{Ca}(2+)$ and GTP binding on tissue transglutaminase tertiary structure. J Biol Chem 275:3915-3921

Eisenberg-Lerner A, Bialik S, Simon HU, Kimchi A (2009) Life and death partners: apoptosis, autophagy and the cross-talk between them. Cell Death Differ 16:966-975

Facchiano F, Facchiano A, Facchiano AM (2006) The role of transglutaminase-2 and its substrates in human diseases. Front Biosci 11:1758-1773

Fesus L, Piacentini M (2002) Transglutaminase 2: an enigmatic enzyme with diverse functions. Trends Biochem Sci 27:534-539

Fesus L, Szondy Z (2005) Transglutaminase 2 in the balance of cell death and survival. FEBS Lett 579:3297-3302

Fesus L, Thomazy V, Autuori F, Ceru MP, Tarcsa E, Piacentini M (1989) Apoptotic hepatocytes become insoluble in detergents and chaotropic agents as a result of transglutaminase action. FEBS Lett 245:150-154

Fimia GM, Piacentini M (2010) Regulation of autophagy in mammals and its interplay with apoptosis. Cell Mol Life Sci 67:1581-1588

Folk JE, Finlayson JS (1977) The epsilon-(gamma-glutamyl)lysine crosslink and the catalytic role of transglutaminases. Adv Protein Chem 31:1-133

Gaudry CA, Verderio E, Aeschlimann D, Cox A, Smith C, Griffin M (1999) Cell surface localization of tissue transglutaminase is dependent on a fibronectin-binding site in its N-terminal betasandwich domain. J Biol Chem 274:30707-30714
Hasegawa G, Suwa M, Ichikawa Y, Ohtsuka T, Kumagai S, Kikuchi M, Sato Y, Saito Y (2003) A novel function of tissue-type transglutaminase: protein disulphide isomerase. Biochem $\mathrm{J}$ 373:793-803

Kahlem P, Green H, Djian P (1998) Transglutaminase as the agent of neurodegenerative diseases due to polyglutamine expansion. Pathol Biol (Paris) 46:681-682

Klionsky DJ (2007) Autophagy: from phenomenology to molecular understanding in less than a decade. Nat Rev Mol Cell Biol 8:931-937

Levine B, Kroemer G (2008) Autophagy in the pathogenesis of disease. Cell 132:27-42

Liu S, Cerione RA, Clardy J (2002) Structural basis for the guanine nucleotide-binding activity of tissue transglutaminase and its regulation of transamidation activity. Proc Natl Acad Sci USA 99:2743-2747

Lorand L, Graham RM (2003) Transglutaminases: crosslinking enzymes with pleiotropic functions. Nat Rev Mol Cell Biol 4:140-156

Luciani A, Villella VR, Esposito S, Brunetti-Pierri N, Medina DL, Settembre C, Gavina M, Raia V, Ballabio A, Maiuri L (2011) Cystic fibrosis: a disorder with defective autophagy. Autophagy 7:92-94

Maiuri MC, Le Toumelin G, Criollo A, Rain JC, Gautier F, Juin P, Tasdemir E, Pierron G, Troulinaki K, Tavernarakis N, Hickman JA, Geneste O, Kroemer G (2007a) Functional and physical interaction between Bcl-XL and a BH3-like domain in Beclin-1. EMBO J 26:2527-2539

Maiuri MC, Zalckvar E, Kimchi A, Kroemer G (2007b) Self-eating and self-killing: crosstalk between autophagy and apoptosis. Nat Rev Mol Cell Biol 8:741-752

Malorni W, Farrace MG, Rodolfo C, Piacentini M (2008) Type 2 transglutaminase in neurodegenerative diseases: the mitochondrial connection. Curr Pharm Des 14:278-288

Malorni W, Farrace MG, Matarrese P, Tinari A, Ciarlo L, MousaviShafaei P, D'Eletto M, Di Giacomo G, Melino G, Palmieri L, Rodolfo C, Piacentini M (2009) The adenine nucleotide translocator 1 acts as a type 2 transglutaminase substrate: implications for mitochondrial-dependent apoptosis. Cell Death Differ 16:1480-1492

Mastroberardino PG, Iannicola C, Nardacci R, Bernassola F, De Laurenzi V, Melino G, Moreno S, Pavone F, Oliverio S, Fesus L, Piacentini M (2002) Tissue' transglutaminase ablation reduces neuronal death and prolongs survival in a mouse model of Huntington's disease. Cell Death Differ 9:873-880

Mastroberardino PG, Farrace MG, Viti I, Pavone F, Fimia GM, Melino G, Rodolfo C, Piacentini M (2006) "Tissue" transglutaminase contributes to the formation of disulphide bridges in proteins of mitochondrial respiratory complexes. Biochim Biophys Acta 1757:1357-1365

Mehta K, Fok JY, Mangala LS (2006) Tissue transglutaminase: from biological glue to cell survival cues. Front Biosci 11:173-185

Melino G, Piacentini M (1998) 'Tissue' transglutaminase in cell death: a downstream or a multifunctional upstream effector? FEBS Lett 430:59-63

Mishra S, Murphy LJ (2004) Tissue transglutaminase has intrinsic kinase activity: identification of transglutaminase 2 as an insulinlike growth factor-binding protein-3 kinase. J Biol Chem 279:23863-23868

Nakaoka H, Perez DM, Baek KJ, Das T, Husain A, Misono K, Im MJ, Graham RM (1994) Gh: a GTP-binding protein with transglutaminase activity and receptor signaling function. Science 264:1593-1596

Oliverio S, Amendola A, Rodolfo C, Spinedi A, Piacentini M (1999) Inhibition of "tissue" transglutaminase increases cell survival by preventing apoptosis. J Biol Chem 274:34123-34128 
Park ES, Won JH, Han KJ, Suh PG, Ryu SH, Lee HS, Yun HY, Kwon NS, Baek KJ (1998) Phospholipase C-delta1 and oxytocin receptor signalling: evidence of its role as an effector. Biochem $\mathrm{J}$ 331:283-289

Pedersen LC, Yee VC, Bishop PD, Le Trong I, Teller DC, Stenkamp RE (1994) Transglutaminase factor XIII uses proteinase-like catalytic triad to crosslink macromolecules. Protein Sci 3:1131-1135

Piacentini M, Colizzi V (1999) Tissue transglutaminase: apoptosis versus autoimmunity. Immunol Today 20:130-134

Piacentini M, Autuori F, Dini L, Farrace MG, Ghibelli L, Piredda L, Fesus L (1991) "Tissue" transglutaminase is specifically expressed in neonatal rat liver cells undergoing apoptosis upon epidermal growth factor-stimulation. Cell Tissue Res 263:227-235

Piacentini M, Farrace MG, Piredda L, Matarrese P, Ciccosanti F, Falasca L, Rodolfo C, Giammarioli AM, Verderio E, Griffin M, Malorni W (2002) Transglutaminase overexpression sensitizes neuronal cell lines to apoptosis by increasing mitochondrial membrane potential and cellular oxidative stress. J Neurochem 81:1061-1072

Rodolfo C, Mormone E, Matarrese P, Ciccosanti F, Farrace MG, Garofano E, Piredda L, Fimia GM, Malorni W, Piacentini M (2004) Tissue transglutaminase is a multifunctional BH3-only protein. J Biol Chem 279:54783-54792

Rubinsztein DC, Gestwicki JE, Murphy LO, Klionsky DJ (2007) Potential therapeutic applications of autophagy. Nat Rev Drug Discov 6:304-312

Szondy Z, Mastroberardino PG, Varadi J, Farrace MG, Nagy N et al (2006) Tissue transglutaminase (TG2) protects cardiomyocytes against ischemia/reperfusion injury by regulating ATP synthesis. Cell Death Differ 13:1827-1829

Tatsukawa H, Fukaya Y, Frampton G, Martinez-Fuentes A, Suzuki K, Kuo TF, Nagatsuma K, Shimokado K, Okuno M, Wu J, Iismaa S, Matsuura T, Tsukamoto H, Zern MA, Graham RM, Kojima S (2009) Role of transglutaminase 2 in liver injury via crosslinking and silencing of transcription factor Sp1. Gastroenterology 136:1783-1795

Tucholski J, Johnson GV (2002) Tissue transglutaminase differentially modulates apoptosis in a stimuli-dependent manner. J Neurochem 81:780-791

Vezza R, Habib A, FitzGerald GA (1999) Differential signaling by the thromboxane receptor isoforms via the novel GTP-binding protein, Gh. J Biol Chem 274:12774-12779

Yamaguchi H, Wang HG (2006) Tissue transglutaminase serves as an inhibitor of apoptosis by cross-linking caspase 3 in thapsigargintreated cells. Mol Cell Biol 26:569-579

Yee VC, Pedersen LC, Le Trong I, Bishop PD, Stenkamp RE, Teller DC (1994) Three-dimensional structure of a transglutaminase: human blood coagulation factor XIII. Proc Natl Acad Sci USA 91:7296-7300

Yousefi S, Perozzo R, Schmid I, Ziemiecki A, Schaffner T, Scapozza L, Brunner T, Simon HU (2006) Calpain-mediated cleavage of Atg5 switches autophagy to apoptosis. Nat Cell Biol 8:1124-1132

Zhang J, Ney PA (2009) Role of BNIP3 and NIX in cell death, autophagy, and mitophagy. Cell Death Differ 7:939-946 